\title{
Acute Pancreatitis with Normal Amylase: A Case Report
}

\author{
Jinous Manouchehri (iD ${ }^{1, *}$ and Yadollah Rashidi ${ }^{1}$ \\ ${ }^{1}$ Emergency Medicine Specialist, Iran University of Medical Sciences, Tehran, Iran \\ "Corresponding author: Emergency Medicine Specialist, Iran University of Medical Sciences, Tehran, Iran. Email: jinous.manouchehri@yahoo.com
}

Received 2021 January 05; Revised 2021 January 28; Accepted 2021 February 06.

\begin{abstract}
Rapid diagnosis in patients with acute pancreatitis is essential to optimal therapeutic outcomes. Upon clinical suspicion, various methods could be used to confirm the diagnosis based on the symptoms and examinations. Laboratory tests are an important diagnostic method in this regard based on the increased serum amylase/lipase. In this study, we described the case of a 31-year-old male patient presenting with the clinical signs of pancreatitis with normal amylase and elevated triglyceride in the follow-up. In case of strong clinical suspicion, other tests and diagnostic methods are recommended to confirm acute pancreatitis.
\end{abstract}

Keywords: Pancreatitis, Amylase, Triglyceride

\section{Introduction}

Acute pancreatitis may lead to severe complications and even significant mortality if it is not diagnosed and treated in a timely manner. Timely diagnostic and therapeutic measures could prevent the complications of the disease in numerous cases. The most common signs and symptoms of acute pancreatitis include abdominal pain, nausea and vomiting, diarrhea, fever, tachycardia, and dyspnea (1). Various diagnostic methods are applied for acute pancreatitis, including laboratory tests, abdominal radiographs, abdominal ultrasound, endoscopic ultrasonography, and CT-scan (2). Increased serum levels of amylase and lipase enzymes are considered to be significant indicators of acute pancreatitis.

In this case study, we described the history of the disease in contrast to the common cases (i.e., normal enzymes) with different radiological evidence supporting acute pancreatitis.

\section{Case Presentation}

Our patient was a 31-year-old man presenting with abdominal pain and two periods of biliary vomiting and food intolerance. The patient's pain was predominantly perceived in the right half of the abdomen and epigastrium. The patient also had a fever and chills and no history of melena, hematemesis, and any specific diseases. However, he had a history of opium and alcohol use.
Baseline vital signs included the blood pressure of 115.65 , pulse rate of 120 , respiratory rate of 30 , and temperature of $38^{\circ} \mathrm{C}$. Initially, the patient was fully conscious and oriented despite appearing restless on physical examination. He also had normal heart sounds without heart murmur, and the lungs had normal hearing as well. There was tenderness on the right abdomen and epigastrium and no organomegaly on the abdominal palpation. Neurological examinations were normal.

\subsection{Laboratory Results}

White blood cell count was 10,800, hemoglobin was 19.6 , platelet count was 104 , blood sugar was 222 , sodium level was 132, potassium level was 4.4 , calcium level was 16.5, liver enzymes were measured to be 58 and 38, creatinine level was 0.5 , sedimentation was estimated at 97, Creactive protein was 48 , total bilirubin was 0.8 , lipase was 88.99 , and amylase level was estimated at 74 .

\subsection{Chest X-Ray}

No specific pathological findings were observed in the lung scan of the patient.

\subsection{Abdominal and Pelvic CT-Scan}

Edema and stranding of pancreatic tissue and peripancreatic showing pancreatitis. A focus of non enhancement seen in trunk of pancreas with volume of $<30 \%$, suggesting necrosis; evidence of acute necrosis seen in spaces of peripancreatic masses and with spread to paracolic gutters on both sides; evidence suggesting necrotizing pancreatitis) (Figure 1). 


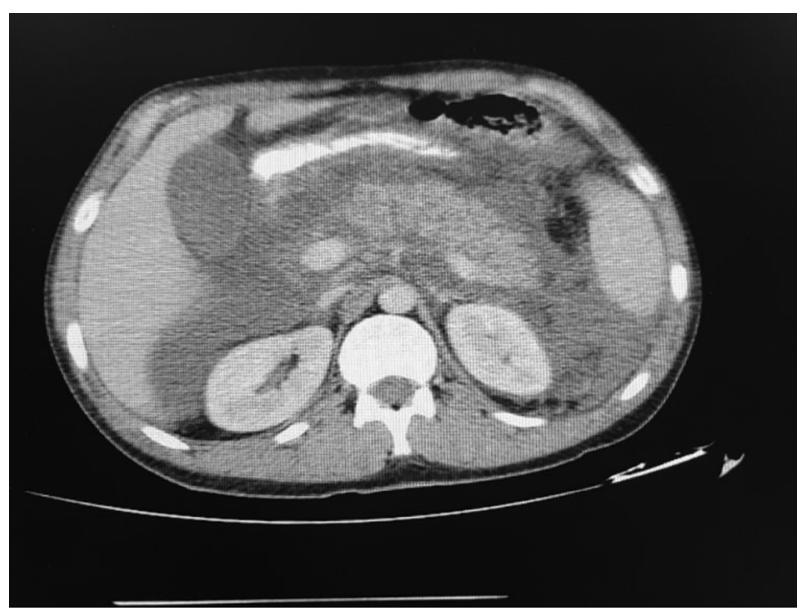

Figure 1. Edema and stranding of pancreatic tissue and peripancreatic showing pancreatitis. A focus of non enhancement seen in trunk of pancreas with volume of $<30 \%$, suggesting necrosis; evidence of acute necrosis seen in spaces of peripancreatic masses and with spread to paracolic gutters on both sides; evidence suggesting necrotizing pancreatitis).

\subsection{Ultrasound}

Ultrasound was performed for the free fluid in the splenorenal space and Morrison's pouch, and the pancreas was observed to be heterogeneous and larger than normal. Furthermore, there was evidence of perivascular inflammation and inflammatory changes around the pancreas, extending to the right anterior parenchyma with the less involvement of the left anterior parenchyma caused by acute pancreatitis. The liver had normal dimensions and echo, the gallbladder was without stones and slug of intrahepatic bile ducts, and common bile duct was normal as well.

\subsection{Subsequent Tests}

As mentioned earlier, triglycerides were measured, and the level was estimated at 1,415 (normal < 150).

\subsection{Patient Procedures and Treatment}

Due to respiratory distress and seizures, the patient was intubated in the emergency section upon admission to the ICU. After eight days, the patient was extubated and transferred to the ward. Notably, the patient had an early admission to the dialysis ward due to aneuria and hypercalcemia, and endocrine counseling was provided for the high triglycerides; no apheresis was required. After stabilization, the patient underwent an endoscopic ultrasound, and after several days of antibiotic treatment, he was discharged in good overall condition and advised to refer to an outpatient clinic after one week.

\section{Discussion}

Laboratory tests are reliable diagnostic approaches for acute pancreatitis. Furthermore, the serum levels of amylase and lipase are significantly elevated in patients with acute pancreatitis, often reaching three times the normal level as observed in the previous studies in this regard (3). Amylase levels in patients with acute pancreatitis may be normal or extremely low due to hypertriglyceridemia or alcohol consumption (4). Previous findings have indicated that normal amylase levels in acute pancreatitis are secondary to hypertriglyceridemia, performing serial amylase test can reduce thetrigyceride interference (5-8).

According to our findings in this case report, the use of other diagnostic methods seems essential to imaging and ultrasound where there is strong clinical suspicion (Figure 2).

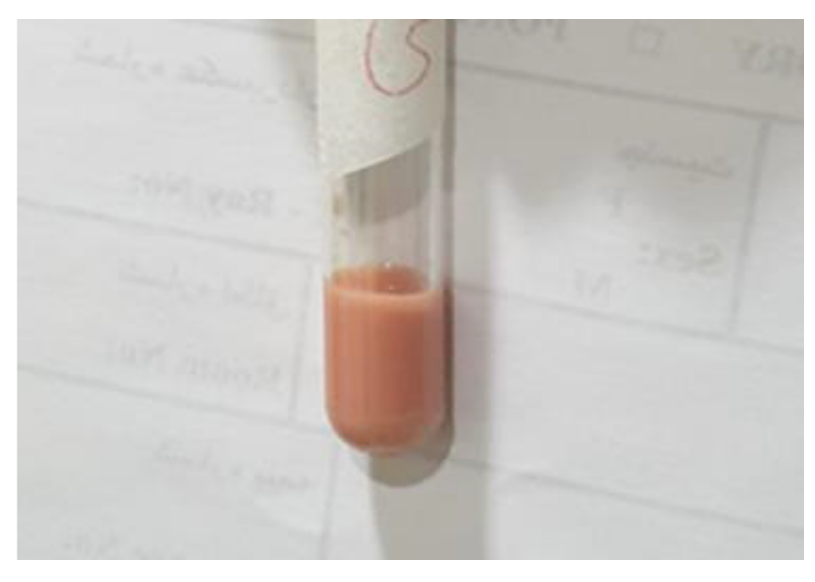

Figure 2. Blood sample of patient after centrifugation

\section{Acknowledgments}

Hereby, we extend our gratitude to professor Dr. Ali Bidari for his valuable contributions to this research project.

\section{Footnotes}

Authors' Contribution: Jinous Manouchehri and Yadollah Rashidi wrote the manuscript.

Conflict of Interests: There was no conflict of interest in current research.

Funding/Support: The authors declare that there is source of funding for the research reported from Iran University of Medical Sciences. 
Informed Consent: Informed consent obtained from patient's parents.

\section{References}

1. Etiology of pancreatitis in united states.In:Acute pancreatitis: Diagnosis and therapy. Bradley; 1994.235 p.

2. Balthazar EJ, Robinson DL, Megibow AJ, Ranson JH. Acute pancreatitis: value of CT in establishing prognosis. Radiology.1990;174(2):331-6. doi: 10.1148/radiology.174.2.2296641. [PubMed: 2296641].

3. Kazmierczak SC. Biochemical Indicators of Acute Pancreatitis. Clinical Pathology of Pancreatic Disorders.1997. p. 75-124. doi:10.1007/978-1-46123964-2_3.

4. Fallat RW, Vester JW, Glueck CJ. Suppression of amylase activity by hypertriglyceridemia. JAMA.1973;225(11):1331-4. [PubMed: 4740657].
5. Yadav D, Agarwal N, Pitchumoni CS. A critical evaluation of laboratory tests in acute pancreatitis. Am J Gastroenterol. 2002;97(6):1309-18. doi: 10.1111/j.1572-0241.2002.05766.x. [PubMed: 12094843].

6. Mathur AK, Whitaker A, Kolli H, Nguyen T. Acute pancreatitis with normal serum lipase and amylase: a rare presentation. J Pancreas. 2016;17(1):98-101.

7. Clavien PA, Robert J, Meyer P, Borst F, Hauser H, Herrmann $F$, et al. Acute pancreatitis and normoamylasemia. Not an uncommon combination. Ann Surg. 1989;210(5):614-20. doi: 10.1097/00000658-198911000-00008. [PubMed: 2479346]. [PubMed Central: PMC1357795].

8. Rao EMM. A Case of Acute Pancreatitis without Enzyme Elevation A Rare Presentation of a Common Condition. Clin Med Rev Case Rep. 2015;2(10). doi: 10.23937/2378-3656/1410066. 